\title{
AN APPLICATION OF VALUATION THEORY TO RINGS OF CONTINUOUS REAL AND COMPLEX-VALUED FUNCTIONS(1)
}

\author{
BY \\ NORMAN L. ALLING
}

Introduction. The presence of an order has played a very prominent role in the study of rings of continuous real-valued functions. Hewitt [17] showed that the residue class fields of these rings are always totally ordered and, over noncompact spaces, may be non-Archimedean. Kohls [22] showed that the residue class domains of these rings are also totally ordered. In some recent researches $[1 ; 2]$ the author has exploited a fact, known to Baer [3] and Krull [23]: that a totally ordered non-Archimedean field has a natural valuation. It is also clear that a totally ordered non-Archimedean integral domain has a natural valuation sorts. These valuations, although derived from the total order on these domains, can be defined for integral domains that are not ordered; thus an attempt at an application of this valuation theory to the residue class domains of the ring of continuous complex valued functions is natural.

The solution of certain integral algebraic equations in these domains will be treated in $\S 1$, together with the proof that these domains are integrally closed in their quotient fields (Theorem 1.8).

In $\$ 2$ a place-like mapping will be defined on these domains, which has many of the algebraic (Theorem 2.2) as well as the topological (Theorem 2.5) properties of a place.

In $\S 3$ an abstract discussion will be given of Abelian groups and integral domains with valuation, followed by an application of these ideas to the natural valuation on the residue class domains of these real and complex function algebras (Theorem 3.6). It will be shown that these valuations are given by the place-like mappings defined in $\S 2$ and can be used to analyze the structure of the prime ideals in these domains in terms of the order and algebraic properties of the value semigroup.

In $\S 4$ order properties akin to that of being an $\eta_{\alpha}$-set will be studied and will be shown to be inherited, in modified form, by the value set of an Abelian group with valuation from the group. Application will then be made to the domains in question (Theorem 4.4).

Received by the editors March 2, 1962.

(1) These researches were done under the support of the National Science Foundation postdoctoral fellowship program at Harvard University. 
In $\S 5$ it will be shown (Theorem 5.1) that a totally ordered Abelian group is a near $\eta_{\alpha}$-set, $\alpha>0$, if and only if certain valuation conditions hold. This result will be used in the proof of Theorem 4.4 and will be used to show (Theorem 5.5) that the domains in question are 1-maximal, a valuation condition.

The order and valuation properties, as applied to the domains under study in this paper, are properties similar to those of an $\eta_{1}$-group. In view of Keisler's recently announced results [19,Appendix], there is accumulating evidence that $\eta_{\alpha}$-set conditions do indeed arise in the residue class fields for $\alpha>1$. That these conditions hold over nondiscrete spaces, that nonmaximal prime ideals exist that are contained in these extraordinary maximal ideals, and that the resulting real domains are near $\eta_{x}$-sets still remains, as far as the author knows, in the realm of wild conjecture. However, the techniques of this paper are sufficiently general to translate any such information about order into information about the structure of the prime ideals in these domains and into information about the degree of maximality of their natural valuations.

1. Let $X$ be a nonempty completely regular Hausdorff space, let $A$ be the ring of all real-valued continuous functions on $X$, and let $A^{\prime}$ be the ring of all complexvalued continuous functions on $X$. Clearly, $A$ is a lattice-ordered algebra over $R$, the reals, and $A^{\prime}$ is an algebra over $C$, the complexes; further, $A$ is a subring of $A^{\prime}$. The absolute value mapping takes $f \in A^{\prime}$ to $|f|$ in $A$. A subset $S^{\prime}$ of $A^{\prime}$ will be called convex in $A$ if, given $s \in S^{\prime}, a \in A^{\prime}$ such that $|a| \leqq|s|$, then $a \in S^{\prime}$. (Throughout this paper whenever an absolute value is present, "convex" will have this meaning.) Let $S^{\prime}$ be a convex subset of $A^{\prime}$; then $S\left(=S^{\prime} \cap A\right)$ is convex in $A$ and $S^{\prime}=\left\{a \in A^{\prime}:|a| \in S\right\}$. Hence $S^{\prime} \rightarrow S\left(=S^{\prime} \cap A\right)$ is a one-to-one order preserving correspondence between the convex subsets of $A^{\prime}$ and those of $A$. Let $A^{\prime *}\left(A^{*}\right)$ be the set of all bounded functions in $A^{\prime}(A)$. Kohls [22] has proved the following proposition for $A$ and $A^{*}$, which generalizes to prove the full result. (See also Gillman and Jerison [11, Theorem 5.5].) By "a prime ideal"' is meant a proper prime ideal.

Proposition 1.1. Prime ideals in $A^{\prime}, A, A^{\prime *}$ and $A^{*}$ are convex.

COROLlaRY 1.2. The prime ideals $P^{\prime}$ of $A^{\prime}\left(A^{\prime *}\right)$ are in one-1o-one order preserving correspondence with the prime ideals $P$ of $A\left(A^{*}\right)$ under the mapping $P^{\prime} \rightarrow P\left(=P^{\prime} \cap A\right)$.

Let $P^{\prime}$ a prime ideal in $A^{\prime}$, let $P=P^{\prime} \cap A$, let $B^{\prime}=A^{\prime} / P^{\prime}$ and let $\delta^{\prime}$ be the canonical homomorphism of $A^{\prime}$ onto $B^{\prime}$. Let $B=\delta^{\prime}(A)$ and let $\delta$ be the restriction of $\delta^{\prime}$ to $A$. Kohls [22] has shown that $B$ may be ordered so that $\delta$ is order preserving and that under this ordering, necessarily unique, $B$ is a totally ordered ring. Every positive element $B$ has an $n$th root in $B$. The following deeper result is known to hold. 
THEOREM 1.3. Any monic polynomial of odd degree with coefficients in $B$ has a root in $B\left({ }^{2}\right)$.

An integral domain $D$ will be called integrally real-closed if it is a totally ordered ring, if every positive element has a square root in $D$, and if every monic polynomial of odd degree with coefficients in $D$ has a root in $D$.

COROLlary 1.4. B is integrally real-closed.

An integral domain will be called integrally algebraically closed if every monic polynomial over it has a root in it: i.e., if every such polynomial splits into linear factors over it.

Proposition 1.5. The quotient field of an integrally real-closed (integrally algebraically closed) integral domain is real-closed (algebraically closed).

Proof. Let $D$ be an integrally real-closed integral domain and let $F$ be its quotient field. Let $F$ be ordered in such a way that the mapping $d \rightarrow d / 1$ of $D$ into $F$ is order preserving; then $F$ is a totally ordered field. Since a positive element $u$ in $F$ may be written as the quotient of positive elements in $D$, each of which has a square root in $D$ (since $D$ is integrally real-closed), $u$ has a square root in $F$. Let $f(y) \in F[y]$ and let it be of odd degree $n$. We may choose $a \in D$, $a \neq 0$, such that $a f(y)=g(y) \in D[y]$. Let the leading coefficient of $g$ be $b$ and let $t=b y$. Then let $b^{n-1} g(y)=h(t)$. Clearly $h(t)$ is a monic polynomial of degree $n$ in $D[t]$. Since $D$ is integrally real-closed, $h$ has a root $d \in D:$ i.e., $d / b$ is a root of $f(y)$ in $F$, showing that $F$ is a real-closed field. A similar argument can be used to prove the remaining statement.

LEMMA 1.6. Let $D$ be an integrally real-closed integral domain that contains a copy of the rational numbers $\left({ }^{3}\right)$. Then $D^{\prime}=D[i]$ is integrally algebraically closed.

Proof. The mapping $i \rightarrow-i$ induces an automorphism on $D^{\prime}$. For $a \in D^{\prime}$ let $\bar{a}$ denote its image under this automorphism. Let $f(y)$ be a monic polynomial with coefficients $a_{j}$ in $D^{\prime}$ and let $\vec{f}(y)$ be the polynomial in $D^{\prime}[y]$ whose coefficients are $\bar{a}_{j}$. Then $f(y) \tilde{f}(y)$ is monic and in $D[y]$; thus it suffices to show that every monic polynomial in $D[y]$ has a root in $D^{\prime}$. Let $f(y)$ be a monic polynomial in $D[y]$ of degree $n=2^{r} s, s$ odd. Since $D$ is integrally real-closed, if $r=0$ then $f(y)$ has a root in $D$. Let $m \in N$ and assume that all monic polynomials $f(y)$ in $D[y]$ have a root in $D^{\prime}$ if $r<m$. Assume that $r=m$.

(2) This theorem is essentially proved by Gillman and Jerison [11, Theorem 13.4], and may be proved by substituting "prime ideal" for "maximal ideal" in their proof. This question has been treated by Hewitt [17], Henriksen and Isbell [16], and Isbell [18].

(3) Some assumption of this sort is necessary, for if $D$ is the set of real algebraic integers then $D$ is an integrally real-closed domain, but $D[i]$ does not contain the roots of $x^{2}+x+1$ and hence is not integrally algebraically closed. 
Let $F^{\prime}$ be the quotient field of $D^{\prime}$ and let $F$ be the subfield of it generated by $D$. By Proposition 1.5, $F$ is real-closed; thus $\mathrm{F}^{\prime}$ is algebraically closed. Let $a_{1}, \cdots, a_{n}$ be the roots of $f(y)$ in $F^{\prime}$. Let $q$ be a rational number and let $b_{j k}=a_{j}+a_{k}+q a_{j} a_{k}$ for $1 \leqq j<k \leqq n$. Let $h(y)=\prod_{1 \leqq j<k \leqq n}\left(y-b_{j k}\right)$. The coefficients of $h(y)$ may be written as polynomials over $D$ in the elementary symmetric functions in $a_{1}, \cdots, a_{n}$, all of which are in $D$, since $f(y) \in D[y]$; thus $h(y) \in D[y]$ and is of degree $n(n-1) / 2=2^{m-1} s\left(2^{m} s-1\right)$. Since $s\left(2^{m} s-1\right)$ is odd, we may invoke the induction hypothesis and conclude that $h(y)$ has a root in $D^{\prime}$. Since the rationals are infinite, there exist $j, k(1 \leqq j<k \leqq n)$ and distinct rational numbers $q$ and $q^{\prime}$ such that $a_{j}+a_{k}+q a_{j} a_{k}=d$ and $a_{j}+a_{k}+q^{\prime} a_{j} a_{k}=d^{\prime}$ are in $D^{\prime}$. Thus $a_{j} a_{k}=\left(d-d^{\prime}\right) /\left(q-q^{\prime}\right)$ and $a_{j}+a_{k} \in D^{\prime}$, and $p(y)=y^{2}-\left(a_{j}+a_{k}\right) y+a_{j} a_{k} \in D^{\prime}[y]$. To show that $f(y)$ has a root in $D^{\prime}$ it suffices to show that any monic polynomial of degree two in $D^{\prime}[y]$ has a root in $D^{\prime}$. Since $\frac{1}{2} \in D$, we may use the quadratic formula to reduce the problem to that of finding a square root in $D^{\prime}$ of every element $z$ in $D^{\prime}$. The imaginary part of $z$ or $\bar{z}$ is non-negative; assume that $z$ has this property and let $z=a+b i, a, b \in D$. Let $c=\left(a^{2}+b^{2}\right)^{1 / 2}$. Clearly $c \in D$ and $c \geqq|a|$. Let $w=((c+a) / 2)^{1 / 2}+((c-a) / 2)^{1 / 2} i$. Since $\frac{1}{2} \in D$ and since $D$ is integrally real-closed, $w \in D^{\prime}$. Clearly $w^{2}=z$ and $(\bar{w})^{2}=\bar{z}$, proving the lemma $\left({ }^{4}\right)$.

Continuing under the hypothesis of Lemma 1.6, we have the following.

COROLlARY 1.7. $D$ and $D^{\prime}$ are integrally closed in their quotient fields.

Proof. Since $D^{\prime}$ is integrally algebraically closed, it is integrally closed in its quotient field $F^{\prime}$. Let $f(y)$ be a monic polynomial in $D[y]$ that has a root $a$ in $F$, the subfield of $F^{\prime}$ generated by $D$, which is of course isomorphic to the quotient field of $D$. Since $D^{\prime}$ is integrally algebraically closed, $f(y)$ splits into linear factors in $D^{\prime}[y]$; thus $a \in D^{\prime} \cap F(=D)$, proving the corollary.

On noting that $B$ contains a copy of the reals, and thus the rationals, we have the following main theorem of the section.

THEOREM 1.8. $\quad B^{\prime}=B[i], B\left(B^{\prime}\right)$ is integrally real-closed (integrally algebraically closed), and is integrally closed in its quotient field, which is real-closed (algebraically closed).

2. Continuing in the notation of the last section, let $O^{\prime}=\left\{b \in B^{\prime}:|b|<n\right.$ for some $n \in N\}$ and let $Q^{\prime}=\left\{b \in B^{\prime}:|b|<1 / n\right.$ for all $\left.n \in N\right\}$. Clearly $O^{\prime}$ is a subring of $B^{\prime}$. Under the canonical homomorphism $\delta^{\prime}$, that maps $A^{\prime}$ onto $B^{\prime}$, the constant functions $C$ in $A^{\prime}$ go into $O^{\prime}$. Further, $Q^{\prime}$ is a prime ideal in $O^{\prime}$ and is the maximal convex subgroup of $O^{\prime}$. Since $\delta^{\prime}\left(A^{\prime *}\right)=O^{\prime}$, we see from Proposition 1.1 , that the prime ideals in $O^{\prime}$ are convex, where $|a+b i|=\left(a^{2}+b^{2}\right)^{1 / 2}$ for $a, b \in B$; thus $Q^{\prime}$ is the maximal ideal in $O^{\prime}$. Let $\pi^{\prime}$ be the canonical homomorphism of $O^{\prime}$ onto $O^{\prime} / Q^{\prime}\left(=k^{\prime}\right)$. Clearly $k^{\prime}$ contains a subfield, $\pi^{\prime} \delta^{\prime}(C)$, isomorphic to thecomplex number field. We will show that $k^{\prime}=\pi^{\prime} \delta^{\prime}(C)$.

(4) This proof is closely related to a proof given by Bourbaki [4, pp. 40-41] on fields. 
Let $O=O^{\prime} \cap A, Q=Q^{\prime} \cap A$, and let $\pi$ be the restriction of $\pi^{\prime}$ to $O . Q$ is a maximal ideal in the ring $O$, it is convex, and further it is the largest proper convex subgroup of $O$. Thus, ordering $k(=\pi(O))$ in such a way that $\pi$ is order preserving, makes $k$ into an Archimedean totally ordered field. $k$ contains $\pi \delta(R)$ as a subfield. Since this subtield is isomorphic to the reals and since $k$ is Archimedean, $k=\pi \delta(R)$. Clearly $O^{\prime}=O(i), Q^{\prime}=Q(i)$, and $k^{\prime}=k(i)$; thus $k^{\prime}=\pi^{\prime} \delta^{\prime}(C)$. We have proved the following lemma.

Lemma 2.1. Given $f \in\left(\delta^{\prime}\right)^{-1} O^{\prime}$ there exists a unique $w \in C$ such that $f-w \in\left(\delta^{\prime}\right)^{-1} Q^{\prime}$.

For $f \in\left(\delta^{\prime}\right)^{-1} O^{\prime}$ let $f^{*}\left(P^{\prime}\right)$ be the complex number $w$ such that $f-w \in\left(\delta^{\prime}\right)^{-1} Q^{\prime}$ and for $f \in A^{\prime}$ and $f \notin\left(\delta^{\prime}\right)^{-1} O^{\prime}$ let $f^{*}\left(P^{\prime}\right)=\infty . f \in A^{\prime}$ will be said to be finite at $P^{\prime}$ if $f^{*}\left(P^{\prime}\right) \in C$ and otherwise infinite at $P^{\prime}$; thus we have the following theorem, which shows that the mapping $f \rightarrow f^{*}\left(P^{\prime}\right)$ has manty of the algebraic properties of a place $\left({ }^{5}\right)$. (See, e.g., Zariski and Samuel [24] for the definition of a place.)

THEOREM 2.2. Let $P^{\prime}$ be a prime ideal in $A^{\prime}$. The mapping $f \rightarrow f^{*}\left(P^{\prime}\right)$ is a homomorphism, of the algebra of elements of $A^{\prime}$ that are finite at $P^{\prime}$, onto the complexes, which is linear over the complexes. If $f \in A, f$ finite at $P^{\prime}$, then $f^{*}\left(P^{\prime}\right)$ is real. Let $f$ be a unit in $A^{\prime}$. $f$ is infinite at $P^{\prime}$ if and only if $(1 / f)^{*}\left(P^{\prime}\right)=0$. Finally, $f$ is finite at $P^{\prime}$ if and only if $a$ and $b$ are finite at $P^{\prime}$, where $f=a+b i$, $a, b \in A ;$ thus $f^{*}=a^{*}+b^{*} i$.

For $f \in A^{\prime}$ let $Z(f)=\{x \in X: f(x)=0\}$ and let $Z\left(P^{\prime}\right)=\left\{Z(p): p \in P^{\prime}\right\}$. (See $[11,2.2]$ for details.) It is easily seen that $Z\left(P^{\prime}\right)$ has the finite intersection property and thus is a directed set (directed down). We now have a result that begins to establish the topological nature of $f^{*}$.

Proposition 2.3. $\operatorname{Lim}_{U \in Z\left(P^{\prime}\right)} f(U)=f^{*}\left(P^{\prime}\right)$; thus $f$ is finite at $P^{\prime}$ if and only if there exists $U \in Z\left(P^{\prime}\right)$ such that $f$ is bounded on $U$.

Proof. Let $f$ be finite at $P^{\prime}$ and let $f^{*}\left(P^{\prime}\right)=w$. Then $\delta^{\prime}(f-w) \in Q^{\prime}$; thus $\left|\delta^{\prime}(f-w)\right|<1 / n$ for all $n \in N$ : i.e., $0 \leqq \delta(|f-w|)<1 / n$ for all $n \in N$. By the nature of the ordering on $B$ (see $[11,5.2]$ ) there exists $p_{n} \in P$ such that $0 \leqq|f-w|+p_{n} \leqq 1 / n$ for all $n \in N$. Let $U_{n}=Z\left(p_{n}\right)$; this is of course in $Z\left(P^{\prime}\right)$. On $U_{n}, 0 \leqq|f-w| \leqq 1 / n$; thus $\operatorname{Lim}_{n \in N} f\left(U_{n}\right)=w$, giving the result if $f$ is finite at $P^{\prime}$. If $f^{*}\left(P^{\prime}\right)=\infty$ then $n<\left|\delta^{\prime}(f)\right|=\delta(|f|)$ for all $n \in N$; thus there exists $r_{n} \in P$ such that $n \leqq|f|+r_{n}$. Let $V_{n}=Z\left(r_{n}\right)$. Clearly $V_{n} \in Z\left(P^{\prime}\right)$ and on $V_{n}, n \leqq|f|$, proving the proposition.

(5) For $f \in A$, given a maximal ideal $M$ in $A, f^{*}(M)$ was defined by Gillman, Jerison and Henriksen [10] using the Stone extension theorem of continuous functions from $X$ to $\beta X$; they derive our definition, as a theorem. The algebraic definition in the real case for prime ideals is considered by Gillman and Jerison [11, Theorem 7.16]. 
Corollary 2.4. Let $M^{\prime}$ be the maximal ideal $[11,2.11]$ of $A^{\prime}$ that contains $P^{\prime}$. Then for $f \in A^{\prime}, f^{*}\left(M^{\prime}\right)=f^{*}\left(P^{\prime}\right)$.

On observing that $Z\left(P^{\prime}\right) \subset Z\left(M^{\prime}\right)$, the result is clear (cf. Gillman and Jerison [11, Theorem 7.16]).

Let $\Sigma$ be the set of all prime ideals of $A^{\prime}$ and let $\Sigma$ be given the hull-kernel topology: i.e., for a nonempty subset $T$ of $\Sigma$, let $\operatorname{kernel}(T)=\bigcap_{P^{\prime} \in T} P^{\prime}$ and for an ideal $I$ in $A^{\prime}$ let hull $(I)=\left\{P^{\prime} \in \Sigma: I \subset P^{\prime}\right\}$. Let $T^{c}$, the closure of $T$ in $\Sigma$, be hull $(\operatorname{kernel}(T))$. Let $\phi^{c}=\phi$. Note: for $P^{\prime} \in \Sigma$, the $\left\{P^{\prime}\right\}^{c}=\left\{P_{1}^{\prime} \in \Sigma: P^{\prime} \subset P_{1}^{\prime}\right\}$ thus $\Sigma$ is not necessarily a Hausdorff space. Let $\Sigma_{0}$ be the set of all maximal ideals of $A^{\prime}$. The topology on $\Sigma_{0}$ induced by $\Sigma$ is the same as the hull-kernel topology on $\Sigma_{0}$, under which it is a compact Hausdorff space.

Let $p \in X$ and let $M_{p}^{\prime}=\left\{f \in A^{\prime}: f(p)=0\right\}$. Clearly $M_{p}^{\prime}$ is a maximal ideal of $A^{\prime}$. Further, $A^{\prime} / M_{p}^{\prime}$ is canonically isomorphic to the complexes. Finally, $f^{*}\left(M_{p}^{\prime}\right)=f(p)$.

THEOREM 2.5. Let $f$ be a fixed element of $A^{\prime}$. The mapping $P^{\prime} \rightarrow f^{*}\left(P^{\prime}\right)$ of $\Sigma$ into the Riemann sphere is continuous. Further, given a continuous mapping $h$ of $\Sigma$ into the Riemann sphere such that $h\left(M_{p}^{\prime}\right)=f^{*}\left(M_{p}^{\prime}\right)$ for all $p \in X$, then $h=f^{*}$.

Proof. It is well known that the mapping $p \rightarrow M_{p}^{\prime}$ is a homomorphism of $X$ onto a dense subset of $\Sigma_{0}$ with which we will identify $X$. There exist unique $a$, $b \in A$ such that $f=a+b i$. Gillman, Henriksen, and Jerison [10] have shown that the Stone extension of $a$ to $\Sigma_{0}$, which is continuous, is (in our terminology) $a^{*} \Sigma_{0}$. We have seen in Theorem 2.2 that $f^{*}=a^{*}+b^{*} i$. Since the finite plane is a topological group, $f^{*}$ is continuous on the set of $M^{\prime} \in \Sigma_{0}$ at which $f$ is finite. If $f^{*}\left(M^{\prime}\right)=\infty$ then $a$ or $b$ is infinite at $M^{\prime}$. Since $|a|,|b| \leqq|f|, f^{*}$ is continuous at $M^{\prime}$, showing that $f^{*}$ is continuous on $\Sigma_{0}$. Let $U$ be a closed set in the Riemann sphere and let $W=\left(f^{*}\right)^{-1}(U)$. Let $P^{\prime} \in W^{c}$ and let $M^{\prime}$ be the maximal ideal of $A^{\prime}$ that contains $P^{\prime}$; then $M^{\prime} \in W^{c}$. Since $f^{*}$ is continuous on $\Sigma_{0}, W \cap \Sigma_{0}$ is closed. Hence $M^{\prime} \in W$ and thus $f^{*}\left(M^{\prime}\right) \in U$. Since $f^{*}\left(P^{\prime}\right)=f^{*}\left(M^{\prime}\right), P^{\prime} \in W$, proving that $f^{*}$ is continuous on $\Sigma$. Clearly $f^{*}$ restricted to $\Sigma_{0}$ is the Stone extension of $f$ to $\Sigma_{0}$, and thus is unique.

Given a continuous extension $h$ of $f$ to $\Sigma$, it must agree with $f^{*}$ on $\Sigma_{0}$. Further, since $M^{\prime} \in\left\{P^{\prime}\right\}^{c}, h\left(M^{\prime}\right)=h\left(P^{\prime}\right)$, proving the uniqueness of $f^{*}$.

We now apply these results to bounded functions to get the following: essentially familiar, result.

Corollary 2.6. Let $f \in A^{\prime} . f$ is bounded if and only if $f$ is finite at $M^{\prime}$ for all maximal ideals $M^{\prime}$ of $A^{\prime}$. Thus, if $f$ is bounded the mapping $P^{\prime} \rightarrow f^{*}\left(P^{\prime}\right)$ is a continuous mapping of $\Sigma$ into the complexes. For $f \in A^{\prime *}$ the mapping $f \rightarrow f^{*}\left(P^{\prime}\right)$ is a linear homomorphism of $A^{\prime *}$ onto $C$.

3. Krull [23] has shown that the prime ideals of the valuation ring of a 
field with valuation are in one-to-one order reversing correspondence with the convex subgroups of the value group. In this section we will develop similar results for Abelian groups and integral domains with valuation, and then apply these results to $B$ and $B^{\prime}$.

By an Abelian group with valuation will be meant a mapping $V$ whose domain $G$ is an Abelian group and whose range is a totally ordered set with a least element, $-\infty$, such that the following conditions hold:

$$
\begin{aligned}
V(a) & =-\infty \text { if and only if } a=0, \\
V(a \pm b) & \leqq \max V(a), V(b) .
\end{aligned}
$$

Let $V$ be an Abelian group with valuation whose domain is $G$. Let $T=V(G)-\{-\infty\}$ be called the value set of $V$. From (3.2) it follows that $V(a) \neq V(b)$ implies $V(a+b)=\max V(a), V(b)$. A subgroup $H$ of $G$ will be called $V$-convex if given $g \in G$ and $h \in H$ such that $V(g) \leqq V(h)$, then $g \in H$. A subset $T^{\prime}$ of $T$ will be called a lower (upper) set of $T$ if given $t \in T$ and $t^{\prime} \in T^{\prime}$ such that $t \leqq t^{\prime}\left(t \geqq t^{\prime}\right)$ then $t \in T^{\prime}$. Note: $\varnothing$ is both an upper set and a lower set of $T$.

Proposition 3.1. The V-convex subgroups of $G$ are in one-to-one order preserving correspondence with the lower sets of $T$ under the mapping $H \rightarrow V(H) \cap T$.

The proof is obvious and is well known in the following special case. Let $G$ be a totally ordered Abelian group. For $a \in G$ let $|a|=\max a,-a$. For $a \in G$ let $V(a)$ be the smallest convex subgroup of $G$ containing $a$. Clearly $\{V(a): a \in G\}$ is totally ordered under inclusion. It is easily verified that $V$ is an Abelian group with valuation. $V$ satisfies the following additional conditions.

$$
\begin{aligned}
& V(a)=V(b) \text { and if only if there exists } n \in N \text { such that }|a| \leqq n|b| \\
& \text { and }|b| \leqq n|a| . \\
& |a| \leqq|b| \text { implies } V(a) \leqq V(b) .
\end{aligned}
$$

Clearly a subgroup of $G$ is convex if and only if it is $V$-convex. $V$ will be called the natural valuation on $G\left({ }^{6}\right)$.

By an integral domain with valuation, $V$, will be meant an Abelian group with valuation whose domain, which in addition to being an Abelian group, is an integral domain and whose value set is a totally ordered semigroup such that the following condition holds:

(6) The idea of a natural valuation on a totally ordered Abelian group, which goes back essentially to Hahn [14], has been used in valuation theory to describe the structure of the value group of a field with valuation, and recently has been used by a number of authors to treat Hahn's theorem. See, e.g., Conrad [7] and Gravett [13]. 


$$
V(a b)=V(a)+V(b) .
$$

Clearly the value set, called the value semigroup, of an integral domain with valuation is commutative and contains an identity element $\left({ }^{7}\right)$. Occasionally we shall refer to $V$ as a valuation on its domain of definition. Clearly an integral domain with valuation is a field with valuation if and only if its domain is a field.

EXAMPLE. Let $S$ be a totally ordered commutative semigroup with an identity. $S$ is said to satisfy the strict monotone condition if given $p, q, r \in S$ such that $p<q$, then $p+r<q+r$. Assume that $S$ satisfies the strict monotone condition. Let $E$ be an integral domain. For $f \in E^{s}$ let $c(f)=\{s \in S: f(s) \neq 0\}$. Let $D=\left\{f \in E^{s}\right.$ : $c(f)$ is finite $\}$. Under pointwise addition $D$ is an Abelian group. For $f \in D, f \neq 0$, let $V(f)$ be the greatest element in $c(f)$. Then $V$ is an Abelian group with valuation. For $f, g \in D$ let $(f g)(s)=\Sigma_{p+r=s} f(p) g(r)$. Under this operation $V$ is an integral domain with valuation, $S$ being the value semigroup of $V$.

Let $D$ be a totally ordered integral domain and let $V$ be its natural valuation, regarding $D$ as only a totally ordered Abelian group. Let $S$ be the value set $V$. Condition (3.5) can be used to define the addition on $S$; thus $V$ is a totally ordered integral domain with valuation which will be referred to as the natural valuation on $D\left({ }^{8}\right)$. It is easily seen that $S$ satisfies the strict monotone condition.

A proper subset $J$ of a semigroup $S$ is called an ideal if $J+S \subset J$, and will be called prime if the set theoretic difference $S-J$ is closed under addition.

Let $V$ be an integral domain with valuation, $D$ its domain of definition, and let $S$ be its value semigroup.

Proposition 3.2. The V-convex prime ideals (ideals) of $D$ are in one-to-one order preserving correspondence with lower sets of $S$ that are prime ideals (ideals) of $S$, under the mapping $I \rightarrow V(I) \cap S$.

This follows from Proposition 3.1 and condition (3.5).

Applying Proposition 3.2, we see that there exists a largest $V$-convex ideal $D_{0}$ of $D$.

Proposition 3.3. $\quad D_{0}$ is a prime ideal of $D$.

Proof. Let $a, b \in D$ such that $a b \in D_{0}$. Assume that $a \notin D_{0}$. Let $D_{0}(a)$ be the ideal of $D$ generated by $D_{0}$ and $a$. Thus $D$ is the $V$-convex subset of $D$ generated by $D_{0}(a)$. Hence there exists $d_{0} \in D_{0}$ and $d \in D$ such that $V(1) \leqq V\left(d_{0}+d a\right)$, and $V(b) \leqq V\left(d_{0} b+d a b\right)$, which is in $V\left(D_{0}\right)$. Since $D_{0}$ is $V$-convex, $b \in D_{0}$, proving the proposition.

If, for example, $D$ is the valuation ring of a field with valuation and if $V$ is the

(7) Here we assume only the weak monotone condition for $S$ : i.e., given $p, q$ and $r$ in $S$ such that $p \leqq q$ then $p+r \leqq q+r$. See Clifford [6] for details.

(8) This idea, which in essence goes back to Hahn [14], was developed by Baer [3] and Krull [23] for totally ordered fields, and for division rings by Conrad [8]. 
restriction of the field valuation to $D$, then $V$ is an integral domain with valuation, and $D_{0}$ is its maximal ideal. This need not always be the case. For example, if $D$ is the polynomial ring in one indeterminate over a field and if $V(f)$ is the degree of $f$, the degree of 0 being $-\infty$, then $V$ is an integral domain with valuation which is not a field and yet $D_{0}=(0)$. Returning to the abstract case, $V$ will be called strong if $D_{0}$ is the maximal ideal of $D$. Let $V\left(D_{0}\right)=S_{0}$.

LEMMA 3.4. If $V$ is a strong integral domain with valuation, then $D_{0}$ is the set of all nonunits of $D, S-S_{0}$ is a group, and $V$ induces a valuation on the field $D / D_{0}$ whose value group is $S-S_{0}$. Further, the $V$-convex prime ideals of $D$ are in one-to-one order reversing correspondence with the upper sets of $S_{0}$ that are closed under addition, under the mapping $I \rightarrow S_{0}-V(I)$.

Proof. Assume that $V$ is a strong integral domain with valuation. Let $d$ be a nonunit of $D$; then $(d)$ is a proper ideal of $D$ and hence is contained in a maximal ideal, namely $D_{0}$. Since $D_{0}$ is a proper ideal, all of its elements are nonunits. $V$ restricted to $D-D_{0}$ maps this multiplicative group homomorphically group onto $S-S_{0}$; thus $S-S_{0}$ is a group. Let $a, b \in D-D_{0}$ such that a $-b \in D_{0}$. Then $V(a)=V(a-b+b)=V(b)$; hence $V$ is constant on the cosets of $D_{0}$ in $D$ other than $D_{0}$ itself, and over these cosets assumes all values in $S-S_{0}$. We conclude that $V$ induces a valuation on $D / D_{0}$ that has $S-S_{0}$ as its value. By Proposition 3.2, it suffices to consider the chain of lower sets $J$ of $S$ that are prime ideals in $S$ in order to establish the last result of the theorem. Let $J$ be a lower set of $S$ that is a prime ideal in $S$; thus $J \subset S_{0}$. Let $L=S_{0}-J$. Since $J$ is a lower set of $S$, it is a lower set of $S_{0}$, and thus $L$ is an upper set of $S_{0}$. Since $J$ is prime, $S-J$ is closed under addition. Since $S_{0}$ is an ideal of $S$, it is closed under addition; thus $L$ is closed under addition. Conversely, let $L$ be an upper set of $S_{0}$ that is closed under addition and let $J=S_{0}-L$. Clearly $J$ is a lower set of $S_{0}$. Since $S_{0}$ is a lower set in $S, J$ is a lower set in $S$. Let $p, q \in S-J$. There exist $r, s \in L$ such that $r \leqq p$ and $s \leqq q$. Thus $p+q \geqq r+s \in L$, proving that $J$ is prime. Let $j \in J$ and $s \in S$. To show that $J$ is an ideal, since $J$ is a lower set, it suffices to show that $j+s \in J$ for $s \geqq 0$. Clearly $s \in S-S_{0}$. Since $S-S_{0}$ is a group, $-s$ exists and is in $S-S_{0}$. Were $j+s \notin J$ then $j+s$ and $-s$ would be in $S-J$. Since $J$ is prime, this would imply that $j \in S-J$, a contradiction; thus $J$ is an ideal. Clearly the mapping $J \rightarrow S_{0}-J$ is one-to-one and order reversing, proving the theorem.

Let $V$ be an integral domain with valuation, let $O_{V}=\{d \in D: V(d) \leqq 0\}$ and $Q_{V}=\{d \in D: V(d)<0\}$. Clearly $O_{V}$ is a $V$-convex subdomain of $D$. Let $V_{0}$ be the restriction of $V$ to $O_{V}$. Then $V_{0}$ is a totally ordered integral domain with valuation whose value semigroup is $S(\leqq 0)(=\{s \in S: s \leqq 0\})$. Clearly $S(<0)$ is a (proper) ideal of $S(\leqq 0)$; thus $Q_{V}$ is, by Proposition 3.2, a prime $V_{0}$-ideal of $O_{V}$, clearly it is the maximal $V_{0}$-convex ideal of $O_{V}$. Let $E=O_{V} / Q_{V}$ and let it be called the residue class domain of $V$. Clearly if $V_{0}$ is strong, then $E$ is a field. 
For $d \in D$ let $d$ be the residue of $d$ in $E$ if $d \in O_{V}$ and let $d=\infty$ if $d \notin O_{V}$. The mapping $d \rightarrow \bar{d}$ of $D^{*}$ onto $E \cup\{\infty\}$ will be called the place associated with $V$.

COROLlary 3.5. Assume that $V$ is a strong integral domain with valuation. Then $V_{0}$ is strong and the residue class domain of $V$ is naturally isomorphic to the residue class field of $D / D_{0}$ under the valuation induced by $V$.

Proof. If $D=O_{V}$ then $S-S_{0}$, which is a totally ordered group, is $\{0\}$; thus $S_{0}=S(<0)$ and $Q_{V}=D_{0}$. Clearly, in this case, the rest of the corollary follows. Assume that $D \neq O_{V}$. Let $a \in O_{V}-Q_{V}$. Since $D_{0} \subset Q_{V}, a$ is a unit in $D$. Let $b$ be its inverse. Then $0=V(1)=V(a)+V(b)=V(b)$; thus $b \in O_{V}$, proving that $V_{0}$ is strong. Clearly $O_{V}$ and $Q_{V}$ are the inverse images of the valuation ring and valuation ideal, respectively, under the canonical homomorphism $D \rightarrow D / D_{0}$, proving the corollary.

Let $V$ be the natural valuation on $B$. Given $w \in B^{\prime}$, there exist unique $a, b \in B$ such that $w=a+b i$. As before, let $|w|=\left(a^{2}+b^{2}\right)^{1 / 2}$. For $f \in A^{\prime}, \delta(|f|)=\left|\delta^{\prime}(f)\right|$, where $\delta$ and $\delta^{\prime}$ are the canonical homomorphisms of $A$ onto $B$ and $A^{\prime}$ onto $B^{\prime}$, respectively. Define $V^{\prime}(w)$ to be $V(|w|)$.

THEOREM 3.6. $V^{\prime}$ is an extension of $V$ and is a valuation of $B^{\prime}$ with the same value semigroup $S . V$ and $V^{\prime}$ are strong; thus $B^{\prime} / B_{0}^{\prime}\left(B / B_{0}\right)$ is naturally isomorphic to $A^{\prime} / M^{\prime}(A / M)$, where $B_{0}^{\prime}\left(B_{0}\right)$ is the maximal ideal of $B^{\prime}(B)$ and $M^{\prime}(M)$ is the maximal ideal of $A^{\prime}(A)$ that contains $P^{\prime}(P)$. The prime ideals of $B^{\prime}(B)$ are in one-to-one order reversing correspondence with the proper upper sets of $S_{0}\left(=V^{\prime}\left(B_{0}^{\prime}\right)=V\left(B_{0}\right)\right)$, that are closed under addition, under the mapping $I \rightarrow S_{0}-V^{\prime}(I)$. For $f \in A^{\prime}$ the mapping $f \rightarrow f^{*}\left(P^{\prime}\right)$ is the same as $\delta^{\prime}$, the canonical homomorphism of $A^{\prime}$ onto $B^{\prime}$, composed with the place associated with $V^{\prime}$.

By Proposition 1.1 the prime ideals in $A^{\prime}(A)$ are convex; thus the prime ideals in $B^{\prime}(B)$ are convex. Clearly the $V^{\prime}(V)$ - convex ideals coincide with the convex ideals in $B^{\prime}(B)$; thus $V^{\prime}(V)$ is strong. The rest of the theorem follows from Lemma 3.4.

4. In order to use Theorem 3.6 to determine the order structure of the prime ideals in $B$, it is necessary to know the order structure of upper sets of $S_{0}$ that are closed under addition. This will be done by considering certain order properties of a totally ordered group that induce similar order properties in its value set.

Let $T$ be a totally ordered set and let $\alpha$ be an ordinal number. $T$ is called an $\eta_{\alpha}$-set (a near $\eta_{\alpha}$-set) if given (nonempty) subsets $T_{0}$ and $T_{1}$ of $T$ of power less than $\aleph_{\alpha}$ such that $T_{0}<T_{1}$, then there exists $t \in T$ such that $T_{0}<t<T_{1}$ $\left(T_{0} \leqq t \leqq T_{1}\right)\left({ }^{9}\right)$. The author has shown [1] that if a totally ordered Abelian

(9) For example, by $T_{0}<t<T_{1}$ we mean that given $t_{0} \in T_{0}$ and given $t_{1} \in T_{1}$ then $t_{0}<t<t_{1}$. The idea of a near $\eta_{1}$-set is known (Kohls [22]); the name and generalization here given appear, however, to be new. 
group (totally ordered field) is an $\eta_{\alpha}$-set, $\alpha>0$, then its value set (value group) is an $\eta_{\alpha}$-set. Combining this result with Krull's theorem, cited at the begining of $\S 3$, and with Proposition 3.1 we arrive at the following.

COROLLARY 4.1. If $\alpha>0$ then the prime ideals of the valuation ring of a totally ordered field that is an $\eta_{\alpha}$-set are in one-to-one order reversing correspondence with the lower sets of an $\eta_{\alpha}$-set.

Erdös, Gillman and Henriksen [9] have shown that a residue class field of $A$ is either real or an $\eta_{1}$-set; in the latter case Corollary 4.1 can be applied.

Gillman and Jerison [11, Theorem 14.15] have shown that $B$ is a near $\eta_{1}$-set. When the next theorem is applied to $B$ in case $P \neq M$, it will show that $S_{0}$ has a property akin to that of being an $\eta_{1}$-set. A nonempty totally ordered set $T$ will be called an upper $\eta_{\alpha}$-set (a lower $\eta_{\alpha}$-set) if given $t_{0} \in T,\left\{t \in T: t>t_{0}\right\}(\{t \in T$ : $\left.\left.t<t_{0}\right\}\right)$ is an $\eta_{\alpha}$-set. $T$ will be called a central $\eta_{\alpha}$-set if given $t_{0}, t_{1} \in T$ such that $t_{0}<t_{1}$, then $\left\{t \in T: t_{0}<t<t_{1}\right\}$ is an $\eta_{\alpha}$-set.

LEMMA 4.2. Let $P$ be a nonmaximal prime ideal in $A$ and let $M$ be the maximal ideal of $A$ that contains $P$. Then $S_{0}\left(=V\left(B_{0}\right)\right)$ is a central $\eta_{1}$-set. If $A / M$ is real, then $S_{0}$ is an upper $\eta_{1}$-set. If $P$ is not the countable intersection of prime ideals that properly contain it, then $S_{0}$ is a lower $\eta_{1}$-set.

Proof. Since $B$ is a near $\eta_{1}$-set, we may apply Theorem 5.1, which tells us that $S$ and $S_{0}$ are central $\eta_{1}$-sets. If $A / M$ is real, then $B_{0}=\{b \in B:|b|<1 / n$ for all $n \in N\}$ and by [11, Theorem 14.16] $S_{0}$ is an upper $\eta_{1}$-set. The final statement is"obvious.

Let $S_{0}$ be a totally ordered commutative semigroup. An upper set of $S_{0}$ that is closed under addition will be called an upper subsemigroup of $S_{0}$. Since the upper subsemigroups of $S_{0}$ are upper sets, they are totally ordered under inclusion. For $r \in S_{0}$ let $W(r)$ be the smallest upper subsemigroup of $S_{0}$ that contains $r$. Then the following hold: $r \leqq s$ implies $W(r) \geqq W(s), W(r)=\left\{s \in S_{0}: n r \leqq s\right.$ for some $n \in N\}, W(r)=W(s)$ if there exist $n, m \in N$ such that $n r \leqq s$ and $m s \leqq r$, and $W(r)<W(s)$ if and only if $s<n r$ for all $n \in N$. Let $T=W\left(S_{0}\right)$ be called the value set of $W$ and let $W$ be called the natural upper valuation on $S_{0}$.

LEMMA 4.3. Let $P$ be a nonmaximal prime ideal in $A$ and let $M$ be the maximal ideal of $A$ that contains $P$. Let $W$ be the natural upper valuation on $S_{0}$ and let $T$ be its value set. Then $T$ is a central $\eta_{1}$-set. If $A / M$ is real, then $T$ is a lower $\eta_{1}$-set, and if $P$ is not the countable intersection of prime ideals that properly contain it, then $T$ is an upper $\eta_{1}$-set.

Proof. Since every positive element of $B$ has an $n$th root in $B$, division by $n$ in $S_{0}$ is always possible, and since $S_{0}$ satisfies the strict monotone condition, the result of this division is unique. Let $T_{1}$ and $T_{2}$ be nonempty subsets of $T$ of power less than $\aleph_{1}$ such that $T_{1}<T_{2}$. For $t \in T$ let $s(t) \in S_{0}$ such that 
$W(s(t))=t$. Let $S_{2}=\left\{(1 / n) s(t): n \in N, t \in T_{2}\right\}$ and let $S_{1}=\left\{n s(t): n \in N, t \in T_{1}\right\}$. Clearly $S_{2}<S_{1}$. Since $S_{0}$ is a central $\eta_{1}$-set, there exists $\mathrm{s} \in S_{0}$ such that $S_{2}<s<S_{1}$. By construction $T_{1}<W(s)<T_{2}$. By similar arguments the rest of the lemma follows.

Combining many of the results of this section and $\S 3$ we get the following theorem.

Theorem 4.4. Let $P$ be a prime ideal in $A, B=A / P, B_{0} P$ the maximal ideal of $B, M$ the maximal ideal of $A$ that contains $P, V$ the natural valuation on $B, S=V(B)$, and $S_{0}=V\left(B_{0}\right) . S_{0}$ is a central $\eta_{1}$-set; thus the prime ideals in $B$ and $B^{\prime}$ are in one-to-one order reversing correspondence with the lower sets of a central $\eta_{1}$-set. If $B / B_{0}(=A / M)$ is real, then $S-S_{0}=\{0\}, S_{0}$ is an upper $\eta_{1}$-set, and the prime ideals of $B$ are in one-to-one order reversing correspondence with the lower sets of an upper $\eta_{1}$-set. If $B / B_{0}$ is not real then $S-S_{0}$ is an Abelian divisible group that that is an $\eta_{1}$-set. If $P$ is not the intersection of a countable family of prime ideals of $A$ that properly contain it, then $S_{0}$ is a lower $\eta_{1}$-set and the prime ideals of $B$ and $B^{\prime}$ are in one-to-one order reversing correspondence with the lower sets of a lower $\eta_{1}$-set.

Kohls [22] (see also Gillman and Jerison [11]) has introduced the following definition: for $a \in B_{0}, a \neq 0$, let $P^{a}$ be the smallest prime ideal in $B$ containing $a$ and let $P_{a}$ be the largest prime ideal in $B$ that does not contain $a$. Then under the correspondence mentioned in Theorem 4.4, $P_{a}$ corresponds to $\{t \in T: t \leqq W V(a)\}$ and $P^{a}$ with $\{t \in T: t<W V(a)\}$. Using Theorem 4.4, one can immediately read off many of Kohls' theorems [22, \$2] and also many of Gillman and Jerison's theorems [11, Chapter 14] on the structure of prime ideals in $A$.

5. In this section necessary and sufficient conditions will be given in terms, of the natural valuation for a totally ordered Abelian group to be a near $\eta_{\alpha}$-set, $\alpha>0$. This result will then be applied to $B$ and $B^{\prime}$.

Let $V$ be an Abelian group with valuation, let $G$ be its domain, and let $T$ be its value set. For convenience let $V(g)$ be identified with $\{x \in G: V(x) \leqq V(g)\}$. For $t \in T$ let $G(t)=\{x \in G: V(x) \leqq t\} /\{x \in G: V(x)<t\}$, and let it be called a factor of $G$. If $G$ is a totally ordered Abelian group and $V$ is its natural valuation, then the factors of $G$ are Archimedean totally ordered groups and hence isomorphic to subgroups of the reals.

Let $S$ be a nonempty totally ordered set and let $S^{\prime}$ be a subset of $S$. $S^{\prime}$ is said to be coinitial (cofinal) in $S$, if given $s \in S$ there exists $s^{\prime} \in S^{\prime}$ such that $s^{\prime} \leqq s$ $\left(s^{\prime} \geqq s\right)$. By the lower (upper) character of $S$ is meant the smallest cardinal number that arises as the cardinal number of a coinitial (cofinal) subset of $S$. If $S$ has no least (greatest) element then its lower (upper) character is an infinite cardinal $\aleph_{\alpha}$. In this case $\omega_{\alpha}$ (the least ordinal, the set of whose predecessors is of power $\aleph_{\alpha}$ ), 
is also referred to as the lower (upper) character of $S$. These are slight modifications of Hausdorff's [15] notion of characters.

Let $T^{\prime}$ be a nonempty upper set of $T$, the value set of $G$, without a least element. Let $G^{\prime}=G /\left\{x \in G: V(x)<T^{\prime}\right\}$. Clearly $V$ induces on $G^{\prime}$ a valuation $V^{\prime}$ that has $T^{\prime}$ as its value set. Taking $\left(\left\{y \in G^{\prime}: V^{\prime}(y)<t\right\}\right)_{t \in T^{\prime}}$ as a fundamental system of open sets of zero in $G^{\prime}$ renders $G^{\prime}$ a topological group; thus it is meaningful to ask if $G^{\prime}$ is Cauchy complete.

Let $\alpha$ be a nonzero ordinal number. An Abelian group with valuation $V$ will be called $\alpha$-maximal if given a nonempty upper set $T^{\prime}$ of $T$, with no least element, whose lower character is less than $\aleph_{\alpha}$ then $G^{\prime}=G /\left\{x \in G: V(x)<T^{\prime}\right\}$ is Cauchy complete. (As observed in [2] this is equivalent to the statement that every pseudoconvergent sequence [20] in $G$ of length less than $\omega_{\alpha}$ has a limit in G.)

In $[1 ; 2]$ the following theorem was proved. Let $G$ be a totally ordered Abelian group, let $V$ be its natural valuation, and let $\alpha$ be a nonzero ordinal number. $G$ is an $\eta_{\alpha}$-set if and only if (i) the factors of $G$ are isomorphic either to the integers or to the reals, (ii) the value set of $V$ is an $\eta_{\alpha}$-set, and (iii) $V$ is $\alpha$-maximal.

The following closely related theorem concerning groups that are near $\eta_{\alpha}$-sets will now be proved.

THEOREM 5.1. Let $G$ be a totally ordered Abelian group, let $V$ be its natural valuation, and let $\alpha$ be a nonzero ordinal number. The following conditions are necessary and sufficient for $G$ to be a near $\eta_{\alpha}$-set:

(i) the factors of $G$ are isomorphic either to the integers or to the reals,

(ii) the value set of $V$ is a central $\eta_{\alpha}$-set,

(iii) $V$ is $\alpha$-maximal $\left({ }^{10}\right)$.

Proof of the necessity of (i), (ii), and (iii). Let $T$ be the value set of $V$ and let $t \in T$. Assume that $G(t)$ is not discrete; then it is isomorphic to a dense subgroup of the real numbers with which we will identify it. Any real number $r$ can be written as the least upper bound (greatest lower bound) of a nonempty countable subset $H(t)(K(t))$ of $G(t)$ that does not contain $r$. Clearly $H(t)<K(t)$. Let $H(K)$ be a set of coset representatives of $H(t)(K(t))$. Clearly $H<K$. Since $G$ is a near $\eta_{\alpha}$-set, there exists $g \in G$ such that $H \leqq g \leqq K$. Clearly $r$ is the image of $g$ in $G(t)$, showing that (i) holds.

Let $T_{0}$ and $T_{1}$ be nonempty subsets of $T$ of power less than $\aleph_{\alpha}$ such that $T_{0}<T_{1}$. For $t \in T$ let $g(t) \in G$ such that $g(t)>0$ and $V(g(t))=t$. Note: $N g\left(T_{0}\right)<g\left(T_{1}\right)$, both sets being nonempty. If $T_{1}$ has no least element, choose $g \in G$ such that $N g\left(T_{0}\right) \leqq g \leqq g\left(T_{1}\right)$. Assume now that $T_{1}$ has a least element $t_{1}$. Further, assume that $G\left(t_{1}\right)$ is nondiscrete. We may choose a descending sequence $\left(g_{n}^{\prime}\right)_{n \in N}$ of positive elements in $G\left(t_{1}\right)$ whose limit is 0 . Let $g_{n}$ be a coset representative of $g_{n}^{\prime}$ in $G$ and choose $g \in G$ such that $N g\left(T_{0}\right) \leqq g \leqq\left\{g_{n}: n \in N\right\}$. Finally,

(10) The proof given here will parallel the proof given of the main theorem in $[1 ; 2]$. 
assume that $G\left(t_{1}\right)$ is discrete. Then $V\left(g\left(t_{1}\right)\right)$ is isomorphic to $Z \times\left\{g \in G: V(g)<t_{1}\right\}$, $Z$ denoting the additive group of integers, the order being lexicographic. Let $\left(g_{s}\right)_{s \in S}$ be a cofinal subset of $\left\{g: V(g)<t_{1}\right\}$. Let $H=\left\{\left(0, g_{s}\right): s \in S\right\}$ and let $K=\left\{\left(1,-g_{s}\right): s \in S\right\}$. Clearly $H<K$. If $|S|<\aleph_{\alpha}$ then, since $G$ is a near $\eta_{\alpha}$-set, there exists $b \in G$ such that $V(b)<t_{1}$ and $H \leqq(a, b) \leqq K, a=0$ or 1 . Note, $a=0$, (1) implies that $g_{s} \leqq b\left(g_{s} \leqq-b\right)$ for all $s \in S$, a contradiction. Hence the upper character of $\left\{g \in G: V(g)<t_{1}\right\}$ is at least $\aleph_{x}$; thus $N g\left(T_{0}\right)$ is not cofinal in it and there exists $g \in G$ such that $N g\left(T_{0}\right)<g$ and $V(g)<t_{1}$. Then, by construction, $T_{0}<V(g)<T_{1}$, showing that (ii) holds: i.e., the value set of $G$ is a central $\eta_{\alpha}$-set.

Let $T^{\prime}$ be a nonempty upper set of $T$, without a least element, whose lower character is less than $\aleph_{\alpha}$. Thus there exists a cofinal subset $\left(t_{\varepsilon}\right)_{\varepsilon<\rho}$ of $T^{\prime}$ such that $\varepsilon<\varepsilon^{\prime}<\rho$ implies $t_{\varepsilon}>t_{\varepsilon^{\prime}} ;$ and $\rho<\omega_{\alpha}$. We must show that $G^{\prime}=G /\left\{g \in G: V(g)<T^{\prime}\right\}$ is Cauchy complete. Thus it suffices to show that any sequence $\left(a_{\delta}^{\prime}\right)_{\delta<\pi}$ in $G^{\prime}$ satisfying the following condition has a limit in $G^{\prime}$ : given $\varepsilon<\rho$ there exists $\delta(\varepsilon)<\pi$ such that $\delta(\varepsilon) \leqq \mu \lambda<\pi$ implies $V^{\prime}\left(a_{\mu}^{\prime}-a_{\lambda}^{\prime}\right)<t_{\varepsilon}$. Let $a_{\delta}$ be a coset representative of $a_{\delta}^{\prime}$ in $G$ and let $b_{\varepsilon} \in G, b_{\varepsilon}>0$, such that $V\left(b_{\varepsilon}\right)=t_{\varepsilon}$. Then $\delta(\varepsilon) \leqq \lambda<\pi$ implies $V\left(a_{\delta(\varepsilon)}-a_{\lambda}\right)<V\left(b_{\varepsilon}\right)$ : i.e., $a_{\delta(\varepsilon)}-b_{\varepsilon}<a_{\lambda}<a_{\delta(\varepsilon)}+b_{\varepsilon}$. Let $H=\left\{a_{\delta(\varepsilon)}-b_{\varepsilon}: \varepsilon<\rho\right\}$ and let $K=\left\{a_{\delta(\varepsilon)}+b_{\varepsilon}: \varepsilon<\rho\right\}$. Clearly $H$ and $K$ are nonempty subsets of $G$ of power less than $\aleph_{\alpha}$. Let $\varepsilon, \varepsilon^{\prime}<\rho$ and let $\lambda$ be an ordinal such that $\delta(\varepsilon), \delta\left(\varepsilon^{\prime}\right) \leqq \lambda<\pi$; then $a_{\delta(\varepsilon)}-b_{\varepsilon}<a_{\lambda}<a_{\delta\left(e^{\prime}\right)}+b_{\varepsilon^{\prime}}$, showing that $H<K$. Since $G$ is a near $\eta_{\alpha}$-set, there exists $g \in G$ such that $H \leqq g \leqq K$ : i.e., if $\delta(\varepsilon) \leqq \lambda<\pi$ then $V\left(a_{\lambda}-g\right) \leqq t_{\varepsilon}$. Then $g^{\prime}$, the image of $g$ in $G^{\prime}$, is the limit of $\left(a_{\delta}^{\prime}\right)_{\delta<\pi}$, showing that (iii) holds.

Assume now that conditions (i), (ii), and (iii) of Theorem 5.1 hold. After proving three lemmas it will be shown that $G$ is a near $\eta_{\alpha}$-set.

By a gap in $G$ (see $\left[15\right.$, p. 90]) is meant a pair $\left(G_{0}, G_{1}\right)$ of nonempty subsets of $G$ such that $G_{0}<G_{1}, G_{0}$ having no greatest element, and $G_{1}$ no least element, such that $G_{0} \cup G_{1}=G$. A gap $\left(G_{0}, G_{1}\right)$ will be called $\alpha$-deficient if the upper character of $G_{0}$ and the lower character of $G_{1}$ are both less than $\aleph_{\alpha}$. Clearly $G$ is a near $\eta_{\alpha}$-set if and only if it has no $\alpha$-deficient gaps.

LEMMA 5.2. If conditions (i), (ii), and (iii) are sufficient in case the value set has no least element, then they are sufficient in case the value set has a least element.

Proof. Assume that the value set $T$ has a least element $t_{0}$ and that (i), (ii), and (iii) hold. Let $T^{\prime}=T-\left\{t_{0}\right\}$. Then $T^{\prime}$ is a central $\eta_{\alpha}$-set whose lower character is at least $\aleph_{\alpha}$. Clearly $U\left(=\left\{g \in G: V(g) \leqq t_{0}\right\}\right)$ is isomorphic to $G\left(t_{0}\right)$ which, by condition (i), is isomorphic either to the reals or to the integers. $V$ induces a natural valuation $V^{\prime}$ on $G^{\prime}=G / U$ which has as its value set $T^{\prime}$. Clearly $G^{\prime}$ satisfies conditions (i), (ii), and (iii). Assume that these conditions imply that $G^{\prime}$ is a near $\eta_{\alpha}$-set. Clearly $G$ is order isomorphic to the set $G^{\prime} \times U$, ordered 
lexicographically. We do not assert that $G$ and $G^{\prime} \times U$ are algebraically isomorphic. Assume that there exists an $\alpha$-deficient gap $\left(C_{0}, C_{1}\right)$ in $G^{\prime} \times U$. Let $G_{i}^{\prime}=\pi_{0} C_{i}, i=0$, 1. Clearly $G_{0}^{\prime}$ and $G_{1}^{\prime}$ are nonempty, $G_{0}^{\prime} \cup G_{1}^{\prime}=G^{\prime}$ and $G_{0}^{\prime} \leqq G_{1}^{\prime}$. Since, by assumption, $G^{\prime}$ is a near $\eta_{\alpha}$-set, it has no $\alpha$-deficient gaps; thus one of the following holds: (1) $G_{0}^{\prime} \cap G_{1}^{\prime} \neq \varnothing$, (2) $G_{0}$ has a greatest element, or (3) $G_{1}$ has a least element. In each case an element $g^{\prime}$ is singled out of $G^{\prime}$. Note: since $T^{\prime}$ has no least element, if (2) and (3) hold, (1) holds; thus $g^{\prime}$ is uniquely determined. Let $U_{i}=\left\{u \in U:\left(g^{\prime}, u\right) \in C_{i}\right\}, i=0,1$. Clearly $U_{0} \cup U_{1}=U$ and $U_{0}<U_{1}$. If both $U_{0}$ and $U_{1}$ are nonempty, then (1) holds. Since $U$ is Dedekind complete (condition (i)) there exists $u^{*} \in U$ corresponding to the cut $\left(U_{0}, U_{1}\right)$ in $U$. Thus $C_{0} \leqq\left(g^{\prime}, u^{*}\right) \leqq C_{1}$, a contradiction since $\left(C_{0}, C_{1}\right)$ is a gap in $G^{\prime} \times U$; thus (2) or (3) hold. Assume that (1) does not hold and that (2) holds. Then $U_{0}$ or $U_{1}$ is empty. Since $g^{\prime} \in G_{0}$ (by (2)), $U_{0} \neq \varnothing$; thus $U_{1}=\varnothing$. Hence $C_{0}=\left\{(x, u): x \in G^{\prime}, u \in U\right.$, and $\left.x \leqq g^{\prime}\right\}$ and $C_{1}=\left\{(x, u): x \in G^{\prime}, u \in U\right.$, and $\left.x>g^{\prime}\right\}$. Clearly the lower character of $C_{1}$ is equal to the lower character of $T^{\prime}$, which is $\aleph_{\alpha}$. Hence $\left(C_{0}, C_{1}\right)$ is not an $\alpha$-deficient gap in $G^{\prime} \times U$, a contradiction. By a similar argument assuming that (1) does not hold and that (3) does hold leads to a contradiction, proving the lemma.

Thus in addition to conditions (i), (ii), and (iii) we may assume the following: (iv) the value set of $V$ does not have a least element.

Thus $G$ is dense. Let $\Sigma$ be the Dedekind completionof $G$ : i.e., let $\Sigma$ be a totally ordered set, without a least, or a greatest, element in which $G$ is imbedded as a dense set and such that any nonempty bounded subset has both a least upper bound and a greatest lower bound. For $a, b \in \Sigma$ let $a L b=1$.u.b. $\{g+h: g, h \in G$, $g \leqq a$, and $h \leqq b\}$ and let $a U b=$ g.l.b. $\{g+h: g, h \in G, g \geqq a$, and $h \geqq b\}$. Clifford [5] has shown that $\Sigma$ is a commutative semigroup under $L$ and $U$. Further, it is easy to see that $a L g=a U g$ for all $g \in G$, the common value being written as $a+g$. The mapping $g \rightarrow-g$ of $G$ onto $G$ induces a mapping $a \rightarrow a^{*}$ of $\Sigma$ onto $\Sigma$. Clearly $a^{* *}=a$ and $(a L b)^{*}=a^{*} U b^{*}$. Let $|a|=\max a, a^{*}$. For $a \in \Sigma$ let $S(a)$ be the smallest convex subset of $\Sigma$ that contains $a$ and is closed under $L .(S(a)$ is also closed under $U$.) Let $W(a)=S(a) \cap G$. The domain of $W$ is $\Sigma$ and its range is a chain of convex subgroups of $G$. Clearly $|a| \leqq|b|$ implies $W(a) \leqq W(b)$, and $W(a L b), W(a U b) \leqq \max W(a), W(b)$. Clearly a gap in $G$ may be uniquely identified with a point in $\Sigma$; such points will be referred to as gaps in $G$.

Lemma 5.3. If $a$ is an $\alpha$-deficient gap in $G, a>0$, then there exist $h, k \in G$, such that $0<h<a<k$ and $V(h)=V(k)$; thus $W(a)=V(h)$.

Proof. Since $a$ is an $\alpha$-deficient gap in $G$ there exist nonempty subsets $H, K$, in $G$ of power less than $\aleph_{\alpha}$ such that 1.u.b. $H=a=$ g.l.b. $K$ and $0<H<K$. Clearly $V(H) \leqq V(K)$. Were $V(H)<V(K)$ we could find $t \in T$ such that $V(H)<t<V(K)$, since $T$ is assumed to be a central $\eta_{\alpha}$-set (condition (ii)). Let 
$g \in G$ such that $g>0$ and $V(g)=t$. Then $H<g<K$, a contradiction since $a$ was assumed to be a gap, proving the lemma.

Thus, if $a$ is an $\alpha$-deficient gap in $G, W(a) \in T$, the value set of $G$. For such $a$, $a-g$ is also an $\alpha$-deficient gap in $G$. In the following lemma it will be shown that $a$ can be approximated by elements of $G$.

Lemma 5.4. If $a$ is an $\alpha$-deficient gap in $G$, then there exists $g \in G$ such that $W(a-g)<W(a)$.

Proof. Let $a$ be an $\alpha$-deficient gap in $G$; thus $a \neq 0$. Clearly $|a|>0$ and is $\alpha$-deficient. Thus we may assume that $a>0$. By Lemma 5.3, there exist $h, k \in G$ such that $0<h<a<k$ and $V(h)=W(a)=V(k)=t$. Let $Y(h)=\{g \in G: V(g)<V(h)\}$. Since $T$ is a central $\eta_{\alpha}$-set, the upper and lower character of $Y(t)$ is at least $\aleph_{\alpha}$. The argument proceeds as in the proof of Lemma 1.4 [2], proving the theorem.

Proof of the sufficiency of conditions (i), (ii), and (iii),. As shown in Lemma 5.2, we may also assume conditions (iv) (i.e., that $T$ has no least element).

Assume that there exists an $\alpha$-deficient gap $a$ in $G$. Let $T^{\prime}=\{t \in T: W(a-g) \leqq t$ for some $g \in G\}$. By Lemma 5.4, $T^{\prime}$ has no least element. Since $a$ is an $\alpha$-deficient gap, the lower character of $T^{\prime}$ is less than $\aleph_{\alpha}$. Let $G^{\prime \prime}=\left\{g \in G: V(g)<T^{\prime}\right\}$. By condition (iii), which we are now using for the first time, $G / G^{\prime \prime}$ is Cauchy complete; thus there exists $g \in G$ such that $W(a-g)<T^{\prime}$, a contradiction, proving Theorem 5.1.

Let $\alpha>0$. Using Hahn group constructions (considered, e.g., in [2]) we can construct a near $\eta_{\alpha}$-group $G$ having an arbitrary central $\eta_{\alpha}$-set $T$ as value set and assuming integral or real factors arbitrarily over $T$. Further, if $|T|=\aleph_{\alpha}$, then such $G$ can be found of power $\aleph_{\alpha}$ (see [2]).

We now apply Theorem 5.1 to $B$ and $B^{\prime}$.

THEOREM 5.5. $B$ and $B^{\prime}$ are 1-maximal; thus if $P\left(P^{\prime}\right)$ is the intersection of a countable number of prime ideals that properly contain it, then $B$ and $B^{\prime}$ are complete in their valuation.

Proof. Gillman and Jerison [11] showed that $B$ is a near $\eta_{1}$-set; thus $B$ is 1-maximal. Given $a, b \in B,|a|,|b| \leqq\left(a^{2}+b^{2}\right)^{1 / 2}$; thus $B^{\prime}$ is 1-maximal, proving the corollary.

Specializing Theorem 5.1 to the case of fields, we have the following.

COROLLARY 5.6. A totally ordered field is a near $\eta_{\alpha}$-set, $\alpha>0$, if and only if

(i') its residue class field is isomorphic to the reals,

(ii') its value group is a central $\eta_{\alpha}$-set,

(iii') it is $\alpha$-maximal.

Let $\alpha>0$. Using formal power series constructions (considered, e.g., in [2]) we can construct a totally ordered field $K$ that is a near $\eta_{\alpha}$-set and that has an arbitrary central $\eta_{\alpha}$-set value group $G$. Further, if $|G|=\aleph_{\alpha}$, such $K$ may be found of power $\aleph_{\alpha}$. Finally, if $G$ is divisible, $K$ can be chosen to be real-closed. 


\section{BIBLIOGRAPHY}

1. N. L. Alling, A characterization of Abelian $\eta_{a}$-groups in terms of their natural valuation, Proc. Nat. Acad. Sci. U.S.A. 47 (1961), 711-713.

2. - On the existence of real-closed fields that are $\eta_{a}$-sets of power $\aleph_{a}$, Trans. Amer. Math. Soc. 103 (1962), 341-352.

3. R. Baer, Über nicht-Archimedisch geordnete Körper, S.-B. Heidelberger Akad. 8 (1927) 3-13.

4. N. Bourbaki, Eléments de mathématique. XIV. II: Algèbre. Actualités Sci. Indust. No. 1179, Hermann, Paris, 1952.

5. A. H. Clifford, Completion of semi-continuous ordered commutative semigroups, Duke Math. J. 26 (1959), 41-59.

6. —_ Totally ordered commutative semigroups, Bull. Amer. Math. Soc. 64 (1958), 305-316.

7. P. Conrad, Embedding theorem for Abelian groups with valuation, Amer. J. Math. 75 (1953), 1-29.

8. —_ On ordered division rings, Proc. Amer. Math. Soc. 5 (1954), 323-328.

9. P. Erdös, L. Gillman and M. Henriksen, An isomorphism theorem for real-fields, Ann. of Math. (2) 61 (1955), 542-554.

10. L. Gillman, M. Henriksen and M. Jerison, On a theorem of Gelfand and Kolmogoroff concerning maximal ideals in rings of continuous functions, Proc. Amer. Math. Soc. 5 (1954), $447-455$.

11. L. Gillman and M. Jerison, Rings of continuous functions, Van Nostrand, Princeton, N. J., 1960.

12. L. Gillman and C. W. Kohls, Convex and pseudoprime ideals in rings of continuous functions, Math. Z. 72 (1960), 399-409.

13. K. A. H. Gravett, Valued linear spaces, Quart. J. Math Oxford Ser. (2) 6 (1955), 309-315.

14. H. Hahn, Über die nichtarchimedischen Grössensysteme, S.-B. Akad. Wiss. Vienna 116 (1907), 601-653.

15. F. Hausdorff, Grundzüg der Mengenlehre, Verlag von Veite, Leipzig, 1914.

16. M. Henriksen and J. R. Isbell, On the continuity of the real roots of an algebraic equation, Proc. Amer. Math. Soc. 4 (1953), 431-434.

17. E. Hewitt, Rings of real-valued continuous functions. I, Trans. Amer. Math. Soc. 64 (1948), 54-99.

18. J. R. Isbell, More on the continuity of the real roots of an algebraic equation, Proc. Amer. Math. Soc. 5 (1954), 439.

19. H. J. Keisler, Ultraproducts and elementary classes, Nederl. Akad. Wetensch. Proc. Ser. A 64 (1961), 477-495.

20. I. Kaplansky, Maximal fields with valuation, Duke Math. J. 9 (1942), 303-321.

21. C. W. Kohls, Ideals in rings of continuous functions, Fund. Math. 45 (1957), 28-50.

22. - , Prime ideals in rings of continuous functions, Illinois J. Math. 2 (1958), 505-536.

23. W. Krull, Allgemeine Bewertungstheorie, J. Reine Angew. Math. 167 (1932), 160-196.

24. O. Zariski and P. Samuel, Commutative algebra, Vol. II, Van Nostrand, Princeton, N. J., 1960.

MassachusetTs Institute of TeChNology,

CAMBridge, MASSACHUSETTS

HARVARD UNIVERSITY,

CAMBridge, Massachusetts

Purdue University,

LAFAYETTE, INDIANA 